\title{
Research on Testing Technology of Aviation Tire Based on Laser Nondestructive
}

\author{
Miao Shang ${ }^{1, a}$ and Min $\mathrm{Ji}^{2, b}$ \\ ${ }^{1,2}$ Xijing University, Shaanxi Xi'an, 710123, China \\ Shaanxi, xi 'an 710065, China \\ aE-mail:2571449451@qq.com, E-mail:995010771@qq.com
}

Keywords: Aircraft Tire; Displacement Speckle; Nondestructive Testing

\begin{abstract}
The article introduces a kind of major nondestructive testing technology about aviation tire - laser digital displacement speckle tires, the working principle of nondestructive testing technology, test equipment, test methods and defect, and adopts the detection technology of manufacturing equipment and its application situation.
\end{abstract}

\section{Introduction}

During take-off and landing, it is important and necessary to conduct the aircraft tire laser nondestructive test, the tires high running speed, and load. If the internal defect exists, it is easy due to the shear strain, flexible heating to produce stress concentration, and the defect is widening. If the defect exists in tread compound, it is easy to happen to jilt tread. And if the defect exists in tire body, flat tire are likely to happen. Both cases are likely to lead to catastrophic accidents plane. NASA's regulations are that civil aviation tire must after nondestructive testing to the factory, also before every renovation to nondestructive testing was carried out on the body. In view of the aviation tire and refurbished aircraft tire, China has respectively established aircraft tire standard (GB9745-1988) and refurbished aircraft tire standards (GB13651-1998), and military aircraft tire standard (GJB108B-1998), including all provisions of nondestructive testing for will check project. Because nondestructive testing is important to avoid the internal defects of tire factory method, it is one of the most effective means to ensure the quality of aircraft tire.

The defects of aviation tire internal are mainly delaminating and bubble, which are fatal defects of aviation tire. Delaminating and bubble exist in the process of the tire manufacturing and use. In the tire manufacturing process of rolling and shaping process, such as between rubber and rubber, tire cord and rubber mixing oil or dirt, or gases between cord and rubber was not fully, these will cause the delaminating and bubbles in inner of tire. After new tires are used for a period of time, glue rickety in tire body interior also can be under the action of shear stress release, and form a new layer. It is almost impossible to detect delaminating and bubbles through routine testing means, but instead through laser nondestructive testing technology. Aviation tire is a nondestructive testing technique that includes laser holographic nondestructive testing technology and laser digital displacement speckle tire nondestructive testing technology. Laser holographic technology applied in aviation tire early NDT, dislocation after development to digital laser speckle technique. This article mainly tells the laser digital displacement speckle technique and its application in aviation tire.

Dislocation laser digital laser speckle tire nondestructive testing technology digital displacement speckle technique is based on the principle of speckle, which uses tire deformation before and after 
the space change of speckle distribution for description of the tire deformation. With the holographic interference detection, dislocation speckle technique is measured by the first derivative of tire deformation, and it is not sensitive to rigid motion. It greatly reduces the environment of the test at the same time, especially the demand of isolation, so that it is applied to the detection technology in the laboratory and ind ustrial environment. It can be widely applied in the on-line measurement of tire production. Its detection principle is shown in Fig. 1.

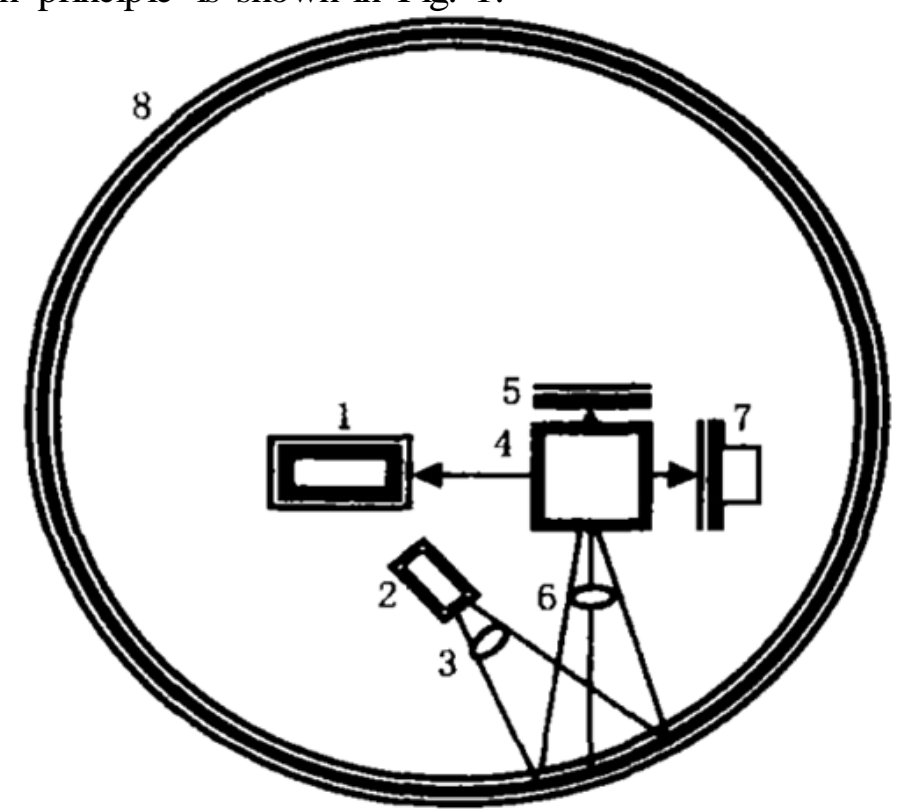

Fig. 1 Dislocation Laser Speckle Detection Principle

1) CCD; 2) Laser;3) Beam Expander;4) Dispersion Prism;5) Mirror;

6) Imaging lens; 7) with Piezoelectric Ceramic Reflector;8) Tires.

Dislocation of defects by speckle detection technology in the detection is based on the comparison of the two deformation object, it can be used with holographic testing in the same way to load of tire, and now it more commonly used is the vacuum load. Dislocation laser digital speckle tire nondestructive testing technology in the detection through high resolution CCD camera system and the precise phase shift technology and phase synchronization algorithm, in the process of stress, the tire changes the strain information real time recorded in the form of phase diagram, and the application of the photoelectric conversion technology will light signal that is converted to digital signals, then calculate and analyze through image processing software and image analysis software, and description of the tire deformation is obtained first derivative of the phase diagram. The distortion by automatic calculation and real-time display parts of the area, and make tires internal defects are detected accurately and clearly displayed on the screen. As shown in Fig. 2 and 3, they are respectively for the tire crown and sidewall parts inspection images. According to Fig. 2 and 3, the defect area and their distribution can be clearly seen.

Dislocation electronic speckle techniques use a variety of digital technology, such as the original photosensitive dry plate into high resolution CCD camera and stored as electronic document, changed both time-consuming and costly, and save and retrieve records complicated optical recording, and well solve the problem of detecting recognition lag. It makes detection, a real-time process for the realization of the paved the way of wide application in industrial environment. 
In terms of detection results analysis and processing, with phase-shift technology, result in the phase diagram of different from the traditional hologram, and it can accurately phase values of each point in the image is presented.

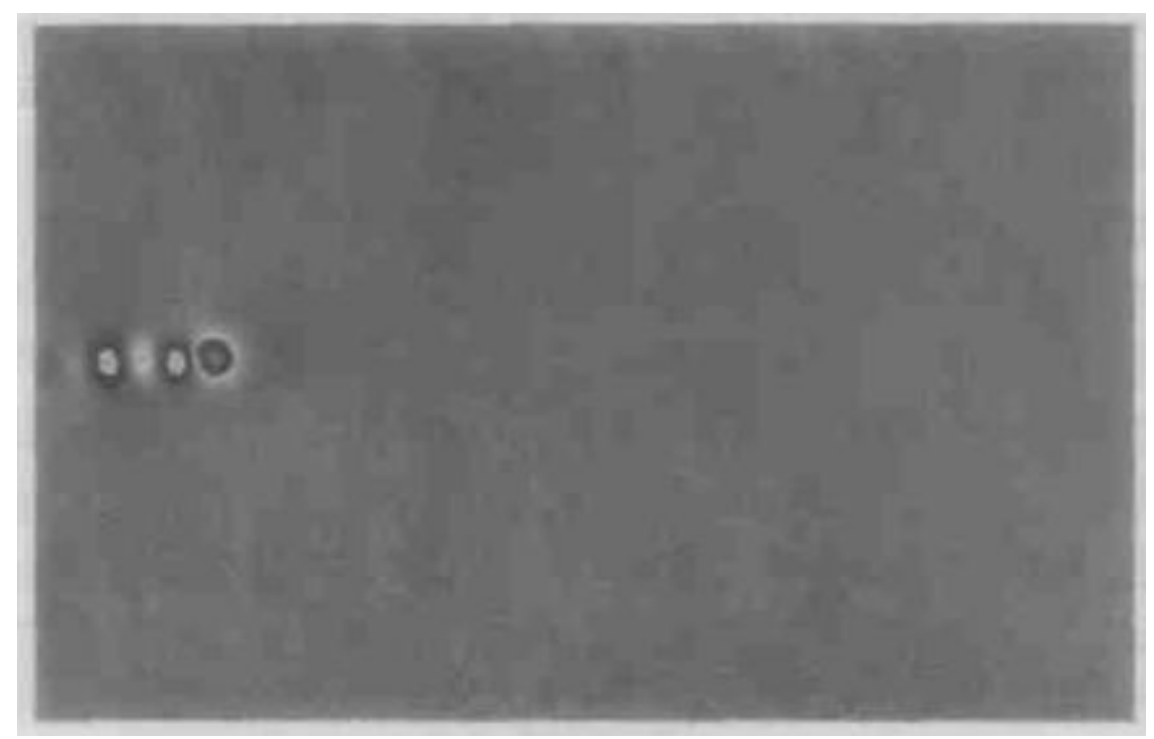

Fig. 2 Laser Displacement Speckle Detection to the Tire Crown Defect Images

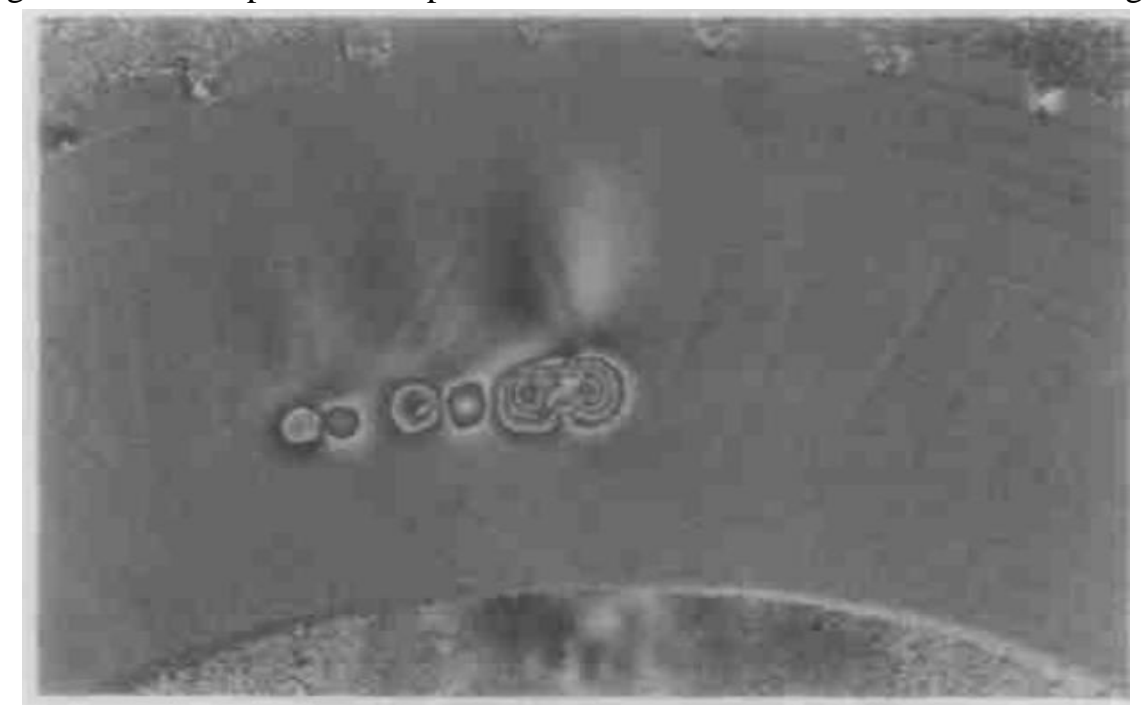

Fig. 3 Dislocation Laser Speckle Detected Sidew all Defect Images

Figure displacement speckle defects detection is the first derivative of the measured surface deformation, interference pattern typical butterfly shaped stripe as shown in Fig. 4, and less affected by the outside world. Although there are many different images from the two tests, in the same way, in the event of a defect, the interference fringes are inevitably existed independently, the circular edge line with normal interference fringes. Circle of stripe said deformation in terms of size, stripe of large deformation; on the other hand, the deformation is small. Distance from the surface at the same time, the defect and is associated with circle in the thickness of stripe, stripe from the defect of coarse surface far, stripe fine defects from the surface. By computer image analysis software is easy to the defects of the tire position and size is calculated. 


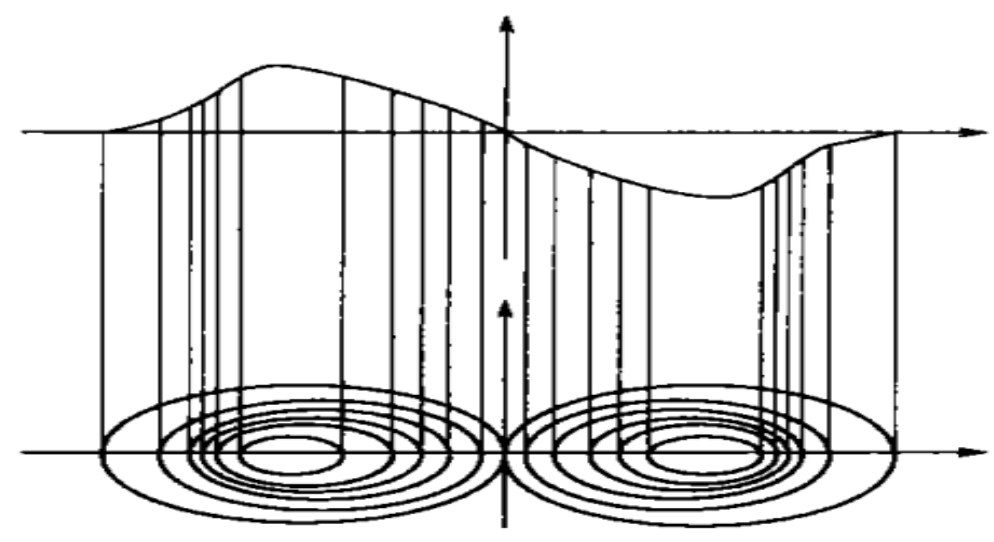

Fig. 4 Displacement Speckle Butterfly Stripes

Laser digital displacement speckle tire nondestructive testing technology is a tire surface condition to the testing of new technology. It is the collection of modern laser technology, speckle interferometry, image acquisition and processing technology, computer technology, precision test technology in the integration of the metrological testing technology. It is characterized by real-time, full contactless condition, the advantages of simple structure and no shockproof device, and it is widely used in the tire production line. Due to the phase shift technology, test results are not affected by the tires of the rigid motion, therefore detector no shockproof device provides a technology base application in the production line. At the same time, the rapid development of semiconductor laser technology makes laser volume smaller, detecting head miniaturization, laser detection head set, beam expander lens, $\mathrm{CCD}$, phase shifter and imaging lens at an organic whole, make the detection head movements freely, and detect any part of tires. Computer image processing and analysis system for the tire defects can be directly displayed on the screen, and the ability to calculate the defect size, intuitive quickly.

\section{References}

[1]ErvinSejdi‘ e1.D.e.JinJiang.Timeequencyfeatuxere Presentationusingenergyconcentration: AnoVer viewofrecentad vanees [J].DigitalSignalProeessing.200919: 153 18.

[2]AbidAliShah, Y.R .Non-destruetivemeasurements of erae kasses smeniand defeedeteetionineonere testructures[J].MaterialsandDesign.2008, 29:61-69.

[3]Hui-xing Yun. Damage detection based-propagation of the longitudinal guided wave in a bimetal composite pipe [J]. Mechanic Automation and Control Engineering (MACE). Hohhot,2011:134-138. 